\title{
Educación media superior obligatoria en México: cobertura y calidad
}

\author{
Compulsory upper secondary education in Mexico: coverage \\ and quality
}
Ensino médio superior obrigatório no México: cobertura e qualidade

Margarita Martínez Martínez* , María de Ibarrola Nicolín**

\section{RESUMEN:}

Este artículo se desprende de una investigación cualitativa en curso. Contrasta el rubro de la reciente obligatoriedad constitucional de la educación media superior, promulgada en el año 2012, contra la cobertura efectiva lograda. Propone un acercamiento al tema a partir del derecho a una "educación de calidad", concepto que se incluye como garantía constitucional, y analiza las estrategias que ha seguido el Estado para alcanzar la cobertura total del nivel, a la vez que se identifican algunos límites que impone el propio Estado para la atención a territorios vulnerables, como municipios y localidades rurales, en los que la oferta de educación media superior (EMS) es precaria o inexistente.

Se desarrolla con base en una revisión documental y en una revisión de las estadísticas más recientes, que se concretan en el análisis del desarrollo de la educación de este nivel y en la oferta educativa en dos localidades del municipio de Tehuitzingo, ubicado en el estado de Puebla.

\section{SUMMARY}

This article is the result of ongoing qualitative research. It contrasts the recent constitutional obligatory nature of higher secondary education, enacted in 2012, against the adequate coverage achieved. It proposes an approach to the subject based
Palabras clave: educación media superior, derecho a la educación, cobertura, calidad.

Key words: higher secondary education, right to education,

\footnotetext{
* Mexicana. Maestra en Ciencias con especialidad en investigación educativa. Centro de Investigación y de Estudios Avanzados del I.P.N. marg_ita@yahoo.com.mx

** Mexicana. Doctora en Ciencias con especialidad en investigación educativa. Centro de Investigación y de Estudios Avanzados del I.P.N. mdeibarrola@gmail.com
} 
on the right to "quality education," a concept that is included as coverage, quality. a constitutional guarantee, and analyzes the strategies followed by the State to achieve total coverage at this level. While at the same time, identifying some limits imposed by the State itself to attend to vulnerable territories, such as municipalities and rural localities, where the offer of higher secondary education (EMS) is precarious or non-existent. It is developed based on a documentary review and most recent statistics, specified in the analysis of education development at this level and in the educational offer in two localities of the municipality of Tehuitzingo, located in the state of Puebla.

\section{RESUMO}

Este artigo é derivado de uma pesquisa qualitativa em curso. Contrasta o título da recente obrigatoriedade constitucional do Palavras-chave: ensino médio superior, direito ensino médio superior, promulgado no ano de 2012, contra a cobertura efetiva conseguida. Propõe uma aproximação do tema a partir do direito a uma "educação de qualidade", conceito que é incluído como garantia constitucional, e analisa as estratégias à educação, cobertura, qualidade. que o Estado tem seguido para conseguir a cobertura total desse nível escolar, ao mesmo tempo que são identificados alguns limites que o próprio Estado impõe para o atendimento de territórios vulneráveis, como municípios e localidades rurais, nos quais a oferta de ensino médio superior (EMS) é precária ou inexistente. É desenvolvido com base em uma revisão documental e em uma revisão das estatísticas mais recentes, que se concretizam na análise do desenvolvimento da educação deste nível e na oferta em educação em duas localidades do município de Tehuitzingo, situado no estado de Puebla. 


\section{Presentación}

Este trabajo forma parte de una investigación cualitativa más amplia -que se encuentra en marcha- sobre la manera como se construyen y se negocian las oportunidades de escolaridad media superior (EMS) en México ${ }^{1}$, cuya universalización no se ha cumplido todavía, a pesar de ser un nivel escolar declarado obligatorio por parte del Estado en el año 2012.

Para este artículo se completó un análisis documental y estadístico minucioso, que permite identificar diversos momentos de la EMS en México, así como dibujar la línea de acción que ha estado siguiendo el Estado para atender el nivel, la consolidación del mismo, la firma de la obligatoriedad y las estrategias de cobertura en diversos contextos. Courrier (1976) considera el análisis documental como esencial, ya que este tipo de análisis pone en contacto al documento con el usuario, por medio de una serie de operaciones intelectuales complejas, cuyo resultado es la representación del documento de una manera condensada y distinta del original, lo que nos permite analizar los elementos más sobresalientes de diversos documentos oficiales que abordan el tema del derecho a la educación, la calidad de la misma y la obligatoriedad del nivel medio superior en México, en documentos como: la Declaración Universal de los Derechos Humanos, la Constitución Política de los Estados Unidos Mexicanos, la Reforma Integral para la Educación Media Superior, la Ley General de Educación, el Programa Sectorial de Educación, el Plan Nacional de Desarrollo, las Principales Cifras del Sistema Educativo Nacional 2019-2020, y diversas investigaciones realizadas por el Instituto Nacional para la Evaluación de la Educación (INEE), entre otros.

El artículo incluye, en un primer momento, los antecedentes del derecho a la educación en México, caracterizado además como el derecho a una educación de calidad. Abordamos el crecimiento, evolución y consolidación del Nivel Medio Superior en México, que se declaró constitucionalmente obligatorio en 2012, y enfatizamos en su situación actual, aprovechando una base de datos estadísticos elab-

1 El sistema escolar mexicano está conformado por tres años de preescolar, seis de primaria, tres de secundaria (educación básica) y tres de educación media superior. Después de este nivel inicia el superior y el posgrado. 
orada por la Subsecretaría de Educación Media Superior en 2018². Este recuento proporciona los elementos para poner dentro del debate la distribución desigual de las oportunidades educativas de la EMS en el país.

Además, identificamos el concepto de la "calidad educativa" como eje rector en cualquier documento que se ha ido emitiendo sobre educación en México en las últimas décadas, y nos sirve como referente para considerar los elementos que la componen y cuáles características permean la educación que llega a los distintos contextos. Ambos análisis se seleccionaron con base en los principios teóricos de política educativa, sobre las brechas y mediaciones entre la promulgación de una política educativa y su implementación (Soberanes, 2015; SEP, 2008; Solís, 2018).

En un segundo momento, hacemos hincapié en las estrategias que ha llevado a cabo el Estado para tratar de alcanzar la cobertura total del nivel en el periodo 2012-2021. Se identifican algunos lineamientos o restricciones que emite el Estado para la oferta del servicio de EMS en contextos vulnerables -localidades y municipios rurales, por ejemplo-, como es el caso de los antes mencionados. Considerando estos elementos, es posible señalar que la oportunidad de acceder al derecho a la educación media superior en México y el tipo de oportunidad que ofrece el Estado están mediados en gran escala por el contexto - urbano o rural- y definidos por la capacidad de presión y negociación de la población.

En un tercer momento se describen las localidades del municipio de Tehuitzingo del Estado de Puebla seleccionadas para el análisis cualitativo completo, de acuerdo con los datos estadísticos que ofrece el Instituto Nacional de Estadística y Geografía (INEGI) y el Consejo Nacional de Población (CONAPO), ya que representan localidades rurales con un alto grado de marginación. Además, permiten ejemplificar la precaria o nula oferta de educación media superior en las localidades así caracterizadas.

2 Elaborada por la Subsecretaría de Educación Media Superior aprovechando el formato 911, que se refiere al instrumento para recopilar información estadística de cada una de las escuelas de todos los niveles educativos en todo el país. Se realiza cada año e incluye número de escuelas, docentes, matrícula, aprobación, reprobación, abandono, para todas las entidades del país y los municipios que las integran. 
En las conclusiones, después del análisis de los alcances de la cobertura, expondremos algunos panoramas sobre el interés y la posición de los actores (habitantes de las localidades) respecto de la oferta de la que disponen y el papel que juega la EMS para los jóvenes que pueden o no cursar el nivel, dada la existencia o no del servicio educativo en el territorio donde viven.

Finalmente, planteamos un panorama de lo que sucede o podría suceder en las localidades donde quedan excluidos del derecho a la educación media superior, tomando en cuenta la capacidad de agencia (Giddens, 1986) de los habitantes de aquellas localidades y la posibilidad de negociar la EMS en lugares donde no existe.

\section{El derecho a la educación per se}

La educación en general es un derecho inalienable, considerado en el país y en todo el mundo.

En el contexto internacional, en la Declaración Universal de los Derechos Humanos el Derecho a la educación está plasmado en el artículo 26, jerarquizando otros derechos por encima de éste. Sin embargo, la educación es considerada como un derecho llave (Latapí, 2009), dado que potencia la exigibilidad y uso de otros derechos, como la libertad de expresión, el acceso a mejores oportunidades laborales, la posibilidad de alcanzar una vida plena y de calidad, entre otros.

En el contexto nacional, la educación se considera un derecho fundamental y una garantía individual. La legislación ha cambiado paulatinamente, así como la estructura, los contenidos y los objetivos educativos para atender la creciente demanda nacional. A lo largo del siglo $\mathrm{XX}$, poco a poco se ha ampliado la estructura educativa que atiende a la población nacional; de la misma manera, se ha trabajado en diferentes legislaciones para lograr que diversos niveles educativos entren en la categoría de educación obligatoria, circunstancia que busca beneficiar a "todos los habitantes de la nación" como directriz general y como eje medular.

En todos los documentos analizados, se enfatiza que la educación pública en México debe ser laica, pública, gratuita y de calidad, con el objetivo de desarrollar la dignidad humana, así como para desarrollar todos los elementos que conlleva. Es importante señalar que la 
obligatoriedad de los distintos niveles educativos en México se estableció en diferentes momentos de la historia, respondiendo a distintas necesidades y a distintas políticas, en orden cronológico: la educación secundaria en 1993; la educación preescolar en el año 2002 y, finalmente, el NMS en $2012^{3}$.

\section{El concepto de "calidad"}

En este punto, es fundamental hacer énfasis en lo que entendemos por "calidad" de la educación, para ello es importante subrayar que el concepto de "calidad", como criterio de valoración de la educación, empezó a circular a fines del siglo pasado, por impulso de los investigadores en educación. Aparece formalmente en los documentos de la transición del poder ejecutivo en el año 2000 y se aplica como tal en los informes del Instituto Nacional para la Evaluación Educativa, compuesto por diversos elementos (INEE, 2000). En 2013 se plasma conceptualmente en la Constitución Política Nacional.

Dada la importancia del INEE (2006) — actualmente MEJOREDU-, retomamos su concepción de la "educación de calidad", pues nos da la pauta para subrayar las características que buscamos encontrar, comparar y analizar en la EMS que ofrece el Estado en los distintos territorios de la república; en este caso, de manera particular y enfática, en las localidades que estamos analizando.

De acuerdo al INEE (2006), una educación de calidad es aquella que reúne los siguientes requisitos:

- en primer lugar esa educación es relevante, porque logra la congruencia del curriculum compartido con las necesidades de la sociedad a la que sirve;

- es pertinente, porque dicho currículo también guarda correspondencia con las necesidades particulares de los alumnos a los que se dirige;

- es internamente, eficaz porque establece una coherencia entre las trayectorias escolares que se dan en los hechos y los objetivos encaminados a lograr que la alta proporción de destinatarios acceda a

3 El cambio constitucional del artículo tercero en el año 2019 establece la obligación del Estado de otorgar Educción Superior a todo el que lo solicite. 
la escuela y permanezca en ella, avanzando con los ritmos previstos hasta su egreso;

- es externamente eficaz porque los logros de aprendizaje se corresponden con los objetivos previstos en el currículo;

- consigue igualmente una correspondencia adecuada entre sus efectos de largo plazo y el objetivo de conseguir la asimilación duradera de los aprendizajes y su traducción en conductas con valor social;

- coordina los procesos y los recursos humanos y materiales, de modo que estos son allegados con suficiencia y son utilizados eficazmente,

- y, en busca de la equidad, ofrece apoyos especiales acordes con las necesidades específicas de quienes lo requieren, para que los objetivos educativos sean alcanzados por el mayor número de estudiantes. (INEE, 2006, p. 16)

Asimismo, podemos añadir en el rubro de educación de calidad algunas características de carácter material y técnico necesarias: instalaciones adecuadas, infraestructura, (aulas, laboratorios, talleres, auditorio), equipamiento suficiente (computadoras), plantilla de profesores suficiente, profesores calificados, manejo adecuado del tiempo (estructuras institucionales del curriculum (De Ibarrola, 2012) y pertinencia de la escolaridad ofrecida (planes, programas, tiempos, espacios, etc.) conjugados con el contexto local. Estos elementos representan la educación de calidad.

\section{La educación media superior en México}

\section{El crecimiento de la EMS en México}

Los esfuerzos por ofrecer escolaridad media superior en México a lo largo del siglo XX permitieron alcanzar $46,8 \%$ de la cobertura del grupo de edad para el año 2012 (SEP, 2012, p. 97). A partir de la firma de la obligatoriedad en esa fecha, el Nivel de Educación Media Superior creció considerablemente e implementó diversas estrategias para alcanzar el 63,6\% de cobertura para 2020 (SEP, 2020, p. 33). Pero la forma en la que lo ha hecho y todas las problemáticas que enfrenta, permiten señalar que no todos los jóvenes logran ingresar al nivel y no todos los que ingresan logran concluirlo. Al margen de lo anterior, es importante señalar que cada nivel educativo tiene sus propias problemáticas y que 
el nivel que ha alcanzado mayor cobertura en México es la educación primaria, como se manifiesta en el Panorama Educativo (2020), con una tasa de cobertura del 98,3\%, mientras que el NMS ha alcanzado el 63,6\%, lo que muestra que está muy lejos de alcanzar la cobertura total nacional.

La bibliografía sobre la desigualdad social, expresada en la distribución de las oportunidades de escolaridad media superior y el acceso a las mismas, es amplia. Arnaut, (2010), Archer (1982) y Villa Lever (2014) enfatizan que el no ingreso a la EMS o el abandono prematuro, suponen un problema de injusticia y de desigualdad social, delimitado no solamente por las desigualdades socioeconómicas entre la población, sino por la ausencia de una política decidida de equidad para la incorporación de los jóvenes a ese nivel.

Con base en estos antecedentes, en este documento ponemos de manifiesto algunos señalamientos e inquietudes sobre la política educativa que afirma la necesidad de establecer la obligatoriedad del nivel medio superior, que conformaría 15 años de educación obligatoria en México ${ }^{4}$, sin tener un plan de acción definido para lograr la cobertura nacional y sin los recursos suficientes ni una estructura establecida para brindar el servicio a todos los jóvenes en edad de cursarlo; aunque cabe señalar que, en la firma de la obligatoriedad, se estableció un periodo de proyección para alcanzar la cobertura total en 2021. Sin embargo, este 2021 -agravado por la pandemia - concluyó el plazo, y la cobertura no ha terminado de llegar a todos los territorios de la república, dejando al margen de este derecho constitucional a los contextos más vulnerables (rurales y marginales). Esta afirmación se sustenta y ejemplifica a partir del análisis de las características de la oferta educativa que presentan las localidades de Los Hornos de Zaragoza y La Noria Hidalgo, del municipio de Tehuitzingo.

Por otra parte, si los avances logrados no permitían vislumbrar una cobertura total en un futuro inmediato, la crisis educativa provocada por la pandemia covid-19 afecta esta posibilidad de manera poco conocida a la fecha.

4 La obligatoriedad de la escolaridad en México ha seguido una línea temporal que parte de la obligatoriedad de la primaria desde mediados del siglo XIX, la secundaria en 1993, el preescolar en el 2002 y, finalmente, el nivel medio superior en 2012. 
Este nivel educativo tiene una larga historia. Surge como parte de la educación universitaria, con la creación de la Escuela Nacional Preparatoria, en 1864, reservada para la elite nacional; en 1934 se funda el Instituto Politécnico Nacional y se crean las escuelas vocacionales de nivel medio superior como preparatorias de ingreso a esta institución. Posteriormente se fue consolidando una amplia gama de subsistemas que ofrece el servicio educativo del nivel medio superior; estos cuentan con historias institucionales disímiles, producto de diversas politicas educativas, modelos curriculares y estrategias institucionales, que marcaron su expansión a lo largo del territorio nacional y que poseen distinto nivel de prestigio entre los jóvenes que demandan el servicio.

La configuración de este nivel educativo, compuesto por diversos subsistemas administrativos, implicó que cada uno de ellos definiera la normatividad operativa en la que se establecieron criterios territoriales para la provisión de estos servicios.

En el periodo de los 70 este nivel tuvo un periodo de auge muy importante, lo que significó incrementar las oportunidades para cursar el nivel más allá de los bachilleratos universitarios y los planteles urbanos; en esa década surgieron diversos subsistemas que ofrecían distintas modalidades y carreras técnicas, vigentes hasta la fecha, como: el Bachillerato Tecnológico Agropecuario (CBTA), el Bachillerato Tecnológico Industrial y de Servicios (CBTIS), los Centros de Estudios Tecnológicos Industrial y de Servicios (CETIS), los Centros de Estudios Tecnológicos del Mar (CETMAR), el Colegio Nacional de Educación Profesional Técnica (CONALEP), el Colegio de Ciencias y Humanidades (CCH), el Colegio de Bachilleres (COLBACH), los Bachilleratos Estatales, los Bachilleratos de Arte del (INBA), los Bachilleratos militares, entre otros.

Una característica fundamental de esa expansión fue la distinción entre instituciones propedéuticas, bivalentes y técnicas terminales; la principal diferencia radicó en el otorgamiento o no del grado académico de bachiller, requisito indispensable para ingresar al nivel superior, y/o del certificado de profesional técnico medio de carácter terminal, sin otorgar los créditos para continuar estudios superiores. Desde ese momento se iniciaron demandas institucionales para que todas las modalidades de educación media superior permitieran la continuidad de los estudios. 
En los 80 llegó un aparente estancamiento. En esta década "perdida" el nivel no solo no creció, sino que los recursos necesarios para su consolidación institucional disminuyeron notablemente. El rasgo de este periodo, fundamentalmente, fue la administración federal del servicio educativo de la EMS a nivel nacional de todos los subsistemas y de todas las modalidades existentes (RIEMS, 2008).

En los 90, la educación media superior vivió un proceso sui generis de descentralización (federalización) ofrecida por el Acuerdo Nacional para la Modernización de la Educación: cada uno de los subsistemas estableció - o no- convenios de descentralización con las entidades federativas y una parte importante de la EMS empezó a ser gestionada por los gobiernos estatales ${ }^{5}$. Los Estados de la república crearon sus propios ODES (Organismos Descentralizados de los Estados); también se creó el Colegio de Estudios Científicos y Tecnológicos del Estado (CECYTE), con lo que la diversidad institucional se incrementó (RIEMS, 2008).

Durante este periodo, este nivel educativo no era considerado obligatorio. De hecho, cada subsistema contaba con sus propias políticas de selección de alumnos. No fue sino hasta el 1996 que se implementó el examen de la Comisión Metropolitana de Instituciones Públicas de Educación Media Superior (COMIPEMS), para el ingreso al NMS en la Ciudad de México y Área Metropolitana. Esta medida se implementó como una política de justicia social y como una estrategia de asignación para los jóvenes demandantes entre el total de instituciones disponibles, dada la amplia gama y diversidad de la oferta institucional en la zona.

En cambio, los jóvenes de contextos rurales difícilmente cuentan con opciones educativas del nivel y deben ceñirse a la precaria, deficiente, o nula oferta que otorga el Estado (educación a distancia, telebachilleratos) y/o a buscar alternativas, si es que desean continuar con sus estudios. En ocasiones, la opción se encuentra en una oferta privada barata y de muy baja calidad (que no es gestionada por el Estado), y que responde a una demanda por el servicio.

En 2008 se puso en marcha la Reforma Integral para la Educación Media Superior (RIEMS), con miras a impulsar la calidad en la enseñan-

5 Los rasgos más representativos de esta gestión fueron el financiamiento estatal de los planteles, nóminas de la plantilla de trabajadores de las instituciones educativas, así como el mantenimiento de las mismas. 
za que ofrece el nivel. La reforma se basó en cuatro pilares: la construcción de un Marco Curricular Común (MCC), la definición y el reconocimiento de todas las opciones de oferta de la EMS, y la profesionalización de los servicios educativos y su certificación nacional. La reforma buscó también unificar los planes de estudio del bachillerato en el país (SEP, 2008); proponía un modelo académico por competencias, en el que la educación estaría centrada en el aprendizaje y no en la enseñanza. La misma reforma propuso la creación de un Sistema Nacional de Bachillerato (SNB), que se encargaría de evaluar la calidad de los planteles de Educación Media Superior. Esto significaba que todas las modalidades institucionales deberían propiciar una educación de calidad como la caracteriza el INEE (2006): relevante, pertinente, eficaz, eficiente, congruente con el contexto y que busca alcanzar la equidad.

En 2012, con el proceso de reforma constitucional, se estableció la obligatoriedad de la educación media superior. Esta reforma trajo consigo la modificación de los artículos $3^{\circ}$ y 31 de la Constitución Política, situación que dio pie a un nuevo periodo de expansión de la EMS e impulsó la necesidad de ampliar la cobertura del nivel a una escala nacional de manera más contundente, para acoger paulatinamente la matrícula nacional y tratar de alcanzar una cobertura total en 2021, (situación que no se ha logrado aún).

\section{Estrategias para alcanzar la cobertura de la EMS}

Desde el momento en que se firmó la obligatoriedad del Nivel Medio Superior en 2012, bajo el gobierno de Felipe Calderón Hinojosa, la problemática actual se gestó, ya que no se contaba con los recursos necesarios, no existían las condiciones económicas y no existía un plan de acción ni la infraestructura para ofrecer a todos los jóvenes el acceso a la EMS; se propuso entonces una posible proyección para alcanzar la cobertura total del nivel para 2021.

Con el paso del tiempo, los siguientes gobiernos asumieron esa obligación heredada y emprendieron una cruzada con diversas estrategias para alcanzar la cobertura nacional. Durante el gobierno de Enrique Peña Nieto (2012-2018) destaca la propagación de la Educación Media Superior a Distancia (EMSAD), la cual no cuenta con una compleja infraestructura, ya que básicamente es una plataforma con contenido educativo modular y tutores en línea; de la misma manera, 
en este periodo creció el número de telebachilleratos estatales y telebachilleratos comunitarios.

Los telebachilleratos funcionan con infraestructura prestada o improvisada. Normalmente, se establecen en instalaciones de telesecundarias o espacios que la comunidad proponga, lo que obliga a trabajar en turnos vespertinos; abren con un mínimo de 12 estudiantes. Se ofrecen 25 horas de clases, de lunes a viernes, cinco por día, con una plantilla de dos o tres docentes, uno de los cuales desempeña al mismo tiempo el cargo de director (con exceso de trabajo, con malas condiciones laborales, rotación constante, etc.). La enseñanza se imparte de manera presencial, se sustenta en el plan de estudios de Bachillerato General, los contenidos se transmiten por televisión y los docentes se apoyan en guías de estudio, que resultan poco significativas para los alumnos y para los contextos en los que operan estas modalidades educativas (INEE, 2018; Guzmán, 2018).

Es fundamental señalar que a, pesar de que se implementa el plan de estudios de Bachillerato General, los telebachilleratos no cuentan con los recursos ni los medios para lograr implementarlo adecuadamente, pues en otros escenarios (urbanos) se asigna un docente para impartir cada asignatura, mientras que en los telebachilleratos se establece una plantilla endeble de docentes para impartir todas las asignaturas. Además, si nos referimos a asignaturas como química o física, no cuentan con laboratorios; si retomamos asignaturas como informática, no tienen equipos de cómputo.

El gobierno actual liderado por López Obrador, en el Programa Sectorial derivado del Plan Nacional de Desarrollo 2019-2024, se compromete a mejorar las condiciones materiales de las escuelas del país, garantizar el acceso de todos los jóvenes y a revertir la "mal llamada reforma educativa”. De la misma manera, la Secretaria de Educación Pública tiene la tarea de dignificar los centros escolares (PND, 2019, p. 51). Asume el compromiso de garantizar el pleno ejercicio del derecho a la educación en todas las regiones del país y para todos los grupos de la población, aunque aún no son muy claras las estrategias que se están siguiendo para lograr la cobertura con calidad del NMS en todo México, y es claro que la pandemia ha generado un impacto negativo, no solo en la ampliación de la cobertura sino en el servicio de por sí ya precario en las localidades rurales. 


\section{Los límites legales al derecho a la educación media superior}

Entendemos que, localmente - alcaldías, municipios, comunidades, localidades, rancherías-, todos estos espacios (pequeños territorios) se rigen por las mismas legislaciones que rigen al país. Conforme con esa lógica, pensando desde documentos como la Constitución Política y la Ley General de Educación, todos los jóvenes (priorizando a los que están en edad de cursar el nivel: 15-17 años) deben recibir educación media superior de calidad, como lo manifiestan los diversos documentos antes citados, con los parámetros que se presentan en el artículo tercero y las características que recuperamos del texto del INEE (2006), sin distinción del contexto, sin priorizar a los jóvenes de las urbes por encima de los habitantes de los territorios rurales, dando a estos últimos opciones que sean equiparables con las que se ofrecen en las ciudades. Vivir en una localidad con pocos habitantes no debería ser una medida de exclusión. Sin embargo, el Estado crea condiciones legales para hacer esa distinción: por una parte, genera una serie de leyes que "deberían amparar a todos los ciudadanos" y, al mismo tiempo, crea reglamentaciones, lineamientos y restricciones que dejan fuera a una fracción importante de la población.

Los lineamientos a los que nos referimos se encuentran plasmados en la normatividad para aperturar un telebachillerato, emitida por la Secretaria de Educación Pública (SEP) y la Dirección General de Bachillerato (DGB), que van dirigidas a pequeñas comunidades y que señalan que la opción que ofrece el Estado a las comunidades que tienen entre 0 a 2.500 habitantes y no cuentan con el servicio de EMS en un radio de $5 \mathrm{~km}$, es el Telebachillerato (DGB, s/f). Sin embargo, es imperativo señalar que el hecho de que, en los lineamientos de la DGB, el telebachillerato sea una opción para las localidades rurales que no tienen una institución de EMS, no significa que estén abriendo uno en cada una de ellas. Por lo tanto, debemos preguntarnos, el derecho a la educación media superior ¿Aplicable a quién? ¿Y bajo qué premisas el Estado priva de la educación que propone como obligatoria y de calidad para todos? ¿Qué elementos permiten o hacen que los contextos rurales sean merecedores de una educación tan precaria o nula? 


\section{Los territorios que estamos analizando...}

Puebla - Tehuitzingo - Los Hornos de Zaragoza y La Noria Hidalgo

\section{El Estado de Puebla}

La entidad se organiza administrativa y políticamente en 217 municipios. Según datos del INEGI (2015), ocupa el quinto lugar de población a nivel nacional; alberga a 6.168.883 habitantes, de los cuales 3.225.206 son mujeres y 2.943.677 hombres.

La principal actividad económica del Estado es la manufactura en materiales textiles y ensamblaje de autos, lo que representa el 3,4 \% de la economía nacional.

Respecto del ámbito educativo, el INEGI (2015) reporta un promedio de 8,5 años de escolaridad para el Estado de Puebla, lo que significa poco más de segundo año de secundaria, por lo que podemos inferir que la población de este Estado no está cerca de alcanzar la cobertura del Nivel Medio Superior.

Adicionalmente, la media estatal no muestra claramente las desigualdades de la educación que se ofrece en las localidades de sus distintos municipios; por ejemplo, las localidades que estamos analizando reportan un promedio de cuatro años de escolaridad entre su población, según datos de la misma fuente.

Tehuitzingo es uno de los 217 municipios que conforman el Estado de Puebla. Cuenta con 11.328 habitantes (INEGI, 2010) y está dividido en 33 localidades, de las cuales cinco están clasificadas en muy alto grado de marginación, 26 en alto grado de marginación y dos en grado de marginación media, según datos de la CONAPO (2015).

Para atender a las 33 localidades del municipio, existen únicamente cuatro planteles de EMS, según la base de datos 911. Por supuesto, uno de ellos se localiza en la cabecera municipal; los otros tres están repartidos en distintas localidades. Estas instituciones ofrecen un servicio educativo precario, que no responde a las necesidades reales de las localidades

Los Hornos de Zaragoza es una de las 33 localidades del municipio de Tehuitzingo. Cuenta con 381 habitantes, según las cifras del INEGI 
(2015). En la localidad hay 184 hombres y 197 mujeres. El promedio de escolaridad es ligeramente superior a cuatro grados de educación (4.82 en hombres y 4.43 en mujeres). Existen 82 analfabetas de más de 15 años; 10 de los jóvenes entre 6 y 14 años no asisten a la escuela; de la población a partir de los 15 años, 67 no cuentan con ningún tipo de escolaridad; 168 cuentan con primaria incompleta, 30 tienen una escolaridad básica y 24 cuentan con educación posbásica (CONAPO, 2015). En la localidad existe un prescolar, una escuela primaria multigrado, una telesecundaria y "NO" existe institución alguna que ofrezca educación media superior.

La Noria Hidalgo es la segunda localidad que estamos analizando. Cuenta con una población total de 920 personas, de las cuales 417 son hombres y 503 mujeres. En esta localidad, según la CONAPO (2015), hay 223 personas analfabetas de 15 años y más, y 16 entre 6 y 14 años no asisten a la escuela. De la población mayor de 15 años, 206 no tienen ninguna escolaridad, 328 tienen una escolaridad primaria incompleta, 71 tienen una escolaridad básica y 76 una educación posbásica.

Cabe señalar que esta localidad es una de las pocas que cuenta con una institución de educación media superior (recordemos que, para las 33 localidades del municipio, solo existen cuatro planteles que ofrecen el servicio): un telebachillerato comunitario, sin instalaciones propias y que utiliza las instalaciones de la telesecundaria a contra turno. Cuenta con un único salón, con mobiliario deficiente e insuficiente, y atiende a 14 alumnos en el turno vespertino (según datos de la base 911).

Ambas localidades están clasificadas en alta marginalidad, situación que las vincula con la desigualdad y la exclusión (CONAPO, 2011). Además, esta situación implica que las condiciones de vida de los habitantes de estas localidades estén regidas por la pobreza (ingresos muy bajos), viviendas sin los servicios mínimos (sin agua potable, sin drenaje, sin luz), muchas veces acompañadas por el hacinamiento; sus condiciones de vida son precarias, no solo en el ámbito educativo, sino en general.

Las actividades económicas que se desarrollan en estas dos localidades están vinculadas básicamente con la agricultura y no con la manufactura, lo que demuestra su aislamiento de las actividades económicas estatales. 
Una característica que no puede pasar inadvertida, es que estas localidades tienen pocos habitantes, lo que podría justificar la ausencia de oportunidades de escolaridad media superior. Sin embargo, son ejemplo de las características de muchas otras localidades del mismo municipio y de muchos otros, clasificados con alta y muy alta marginalidad, por lo que, si se suman a todos los jóvenes habitantes de todas las localidades que se encuentran en estas condiciones, el número alcanza una proporción elevada del grupo de edad (15-17 años), y esto no puede ser considerado como un asunto menor. Son muchos los jóvenes que quedan excluidos del derecho a la EMS y claramente se desdibuja el concepto de "calidad", ya que las características que presenta la institución de NMS ubicada en la localidad de La Noria Hidalgo están muy lejos de alcanzar los estándares que plantea el INEE (2006): dicha institución no cuenta con los requisitos mínimos, no es pertinente, no es eficaz, no es eficiente, no es congruente con el contexto, no ayuda a erradicar la desigualdad y no cuenta con infraestructura propia ni recursos suficientes.

En el caso de la localidad de Los Hornos de Zaragoza el problema es más complicado, ya que no hay nada que medir o comparar, simplemente no existe institución alguna que ofrezca el servicio del nivel y los jóvenes de la localidad quedan excluidos de este derecho. Si desean continuar sus estudios, deberán buscar algunas alternativas, como acudir a otra localidad a estudiar, si es que cuentan con los recursos económicos suficientes, de lo contrario no podrán continuar con su formación académica.

Tabla 1.

Planteles de educación media superior en el Municipio de Tehuitzingo

\begin{tabular}{llll}
\hline Estado de Puebla 21 Tehuitzingo, Municipio 157 & & \\
\hline $\begin{array}{l}\text { Institución de educación media } \\
\text { superior }\end{array}$ & $\begin{array}{l}\text { Suma de } \\
\text { alumnos } \\
\text { Total }\end{array}$ & $\begin{array}{l}\text { Suma de } \\
\text { egresados } \\
\text { total }\end{array}$ & $\begin{array}{l}\text { Suma de } \\
\text { docentes } \\
\text { Total }\end{array}$ \\
\hline CECYTE 21ETC0012Y & 273 & 76 & 15 \\
\hline OCES 21EBH0438H & 68 & 17 & 9 \\
\hline OCES 21EBH0606N & 60 & 18 & 3 \\
\hline $\begin{array}{l}\text { Telebachillerato comunitario } \\
\text { 2letk0003z }\end{array}$ & 14 & 6 & 2 \\
\hline Total general & 415 & 117 & 29 \\
\hline
\end{tabular}

Fuente: Elaboración propia con datos de la base de datos 911 (2018). 


\section{Conclusión}

Agencia y negociación. ¿Qué pasa cuando no hay escuelas de EMS?

Frente al hecho de quedar excluidas del derecho a la EMS, estas localidades tienen capacidad de agencia. Pueden -o no- llevar a cabo distintas estrategias para ser incluidas, y en estos territorios se pueden gestar distintos escenarios con la participación de diferentes actores ante la oportunidad de acceder al derecho a la educación. Un factor determinante es el sentido que este último les inspira o la percepción que tienen de la escuela (sentido de la escuela) (Weiss, 2012). Dependiendo del nivel de significado que les genere la educación, ellos actuarán o no para conseguirla.

Después de varios análisis, proponemos que estos son los posibles escenarios:

a) el territorio está conforme con la oferta que le brinda el Estado (¿ni modo?);

b) no existe interés por parte del territorio para exigir su derecho a la EMS;

c) el territorio pondera la importancia de su derecho a la educación media superior y los actores que son parte del territorio se organizan y actúan para demandar y exigir el servicio educativo;

d) los habitantes, en la individualidad, resuelven la insuficiencia o ausencia de oportunidades educativas del nivel.

En este estudio, concebir a las localidades como territorios refiere no solo al espacio físico que ocupan; incluye la presencia de habitantes y gobierno en ellas, dinámicas y actividades económicas, política, sociales y culturales (Sandoval, 2017). Por un lado, entonces, existe una fracción que genera las leyes de educación y en cierto sentido las restricciones, pero los habitantes (individuos) tienen capacidad de acción, "capacidad de agencia" como la designa Giddens (1986). La agencia es la capacidad de los seres humanos para tomar decisiones y actuar con la intención de producir un efecto; es la habilidad de actuar de manera intencional, de actuar con un propósito. La acción y las decisiones están basadas en una interpretación de la situación sobre la que se va a actuar y, si la educación les significa algo importante, al te- 
ner un posible escenario los individuos actúan y, si es necesario, se organizan: es lo que conocemos como "capacidad colectiva". Esta noción (Murphy, 2014) refiere a la capacidad central de una sociedad o institución para decidir sobre su futuro. Lo más importante es la dimensión política colectiva ya que, a partir de esta perspectiva, se estructurarán las instituciones sociales, y es donde tiene cavidad la acción del colectivo, la negociación y la presencia de diversos actores locales.

Entonces, independientemente de las restricciones que imponga el Estado, si existe interés en la educación por parte de los pobladores de las localidades, estos buscarán estrategias para alcanzar el derecho a la educación por medio de lo que denominaremos "negociación". Existen diversos antecedentes de que esta práctica se ha llevado a cabo en países como Argentina (Cerletti, 2010; Gentilini, 2011); Chile (Juárez, 2016); Colombia (Aries, 2017); Perú (Ames, 2011), entre otros, y aparecen en ella diversos actores, como intelectuales, maestros, empresarios, representantes de la administración pública, padres de familia, estudiantes, entre otros. En este proceso de negociación, representantes de las localidades y del gobierno trataran de llegar a acuerdos en pro del derecho a la educación.

\section{Lo que se negocia...}

En este caso, el sentido de la negociación es la obtención del derecho a la EMS y el ejercicio pleno del mismo, o de lograr mejoras educativas en el NMS que beneficien a los habitantes. La negociación se refiere a los puntos de inflexión, las concesiones que realizan en principio dos grandes grupos de interés: los representantes del gobierno federal, estatal, municipal o local, los representantes de la administración pública, por una parte, y los habitantes de las localidades, por otra, y se refiere también a los puntos en que se puede aprobar o ceder.

En los procesos de negociación se puede pactar sobre distintos elementos. Desde la misma educación media superior, ¿cómo se negocian la EMS y cada uno de los elementos de la calidad (pertinencia, eficacia, relevancia, entre otras)? ¿Qué concesiones hace cada actor? ¿Quién decide qué subsistema y modalidad cubrirá la oferta? Y, si es el caso de una modalidad técnica o tecnológica, ¿qué carreras se impartirán? ¿Qué turnos se trabajarán? ¿Quién dona el terreno? ¿Quién edifica la infraestructura y le da mantenimiento a las instalaciones? ¿Quién 
contrata a los maestros y bajo qué condiciones laborales? ¿Cómo intervienen las empresas locales? ¿Quién realiza la vinculación? ¿A qué distancia? ¿Cómo se resuelve la transportación de los alumnos?, entre otras. También sería importante revisar cuánto tiempo tarda una negociación.

Es posible afirmar que, en los contextos descritos, no ha existido un amplio proceso de negociación y los frutos son escuetos; hasta el momento existen cuatro planteles de EMS en cuatro de las 33 localidades del municipio de Tehuitzingo y son insuficientes para atender a los jóvenes en edad de cursarlo; tampoco existe un panorama claro de cuándo cambiará la situación. Al parecer tres bachilleratos estaban consolidados antes de la firma de la obligatoriedad y en nueve años (2012-2021), a partir de la cruzada de la cobertura, solamente se abrió el telebachillerato ubicado en la localidad de La Noria Hidalgo, pero continúa siendo insuficiente.

Las consecuencias que traen consigo las limitaciones o la negación en los hechos del derecho a la educación son muchas; entre ellas, pocas oportunidades educativas para los jóvenes, que se traducen en rezago educativo, pobreza, migración, entre otros.

Finalmente, es fundamental alcanzar la cobertura nacional total del nivel, pero también es importante ofrecer la calidad educativa que tanto se presume en los distintos documentos, a todos los contextos, sin distinción, ya que ningún grupo o sector debería ser excluido de ese tipo de educación que el Estado "promueve", ya que, como menciona Dubet (1998), para unos el bachillerato sanciona una pertenencia a un contexto de pobreza, como pasa con los jóvenes de las múltiples localidades que existen en México, clasificadas con alto y muy alto nivel de marginación, que difícilmente escaparán del círculo de la pobreza; mientras que, para otros, la educación asegura una movilidad y una mejor calidad de vida.

\section{Bibliografía}

Ames (2011). La educación rural. Balance de cinco décadas de estudio. Lima Perú: SEPIA.

Comisión Metropolitana de instituciones Públicas de Educación Media Superior COMIPEMS. (2020). Recuperado de https:// www.dgae.unam.mx/noticias/examunic/menu.html 
CONAPO. (2011). Concepto y dimensiones de la marginación. Recuperado de http://www.conapo.gob.mx/work/models/CONAPO/Resource/1755/1/images/01Capitulo.pdf

Courrier, Y. (1976). Analyse et language documentaire. Docurnentaliste, 13(5-6), 178.

Cragnolino, E. (2017). Desde la escuela primaria a la escuela secundaria campesina. Luchas por la educación pública en Córdoba Argentina. Educ. Soc. [online], 38(140), 671-688.

DUDH. (2015). Declaración Universal de los Derechos Humanos, Naciones Unidas. Recuperado de https://www.un.org/es/documents/udhr/UDHR_booklet_SP_web.pdf

De Ibarrola, M. (Coord.) et al. (2018) Los desafíos que enfrenta la formación de los jóvenes para el trabajo del siglo XXI. Las escuelas de nivel medio superior y otras alternativas. Informe de investigación entregado a la Subsecretaría de EMS, noviembre de 2018.

Diario Oficial de la Federación (DOF). (1917). Constitución Política de los Estados Unidos Mexicanos, última reforma, 29 de enero de 2016. México: Cámara de diputados. Recuperado de http://www.diputados.gob.mx/LeyesBiblio/htm/1.htm

Diario Oficial de la Federación (DOF). (2002). Obligatoriedad de la educación preescolar. Recuperado de http://dof.gob.mx/index.php?year $=2002 \&$ month $=12 \&$ day $=30$

Diario Oficial de la Federación (DOF). (1917). Ley General de educación. Revisado el 1 de junio, 2020.

Dirección General de Bachillerato. (2014). Telebachillerato Comunitario. Recuperado de https://www.gob.mx/sep/acciones-yprogramas/telebachillerato-comunitario?state=published

Dubet, F. (1998). En la escuela. Sociología de la experiencia. España: Editorial Losada.

Giddens, A. (1986). The constitution of society. Cambridge: Polity Press.

Gobierno de México. (2019). Plan Nacional de Desarrollo 2020-2024. Recuperado de https://framework-gb.cdn.gob.mx/landing/ documentos/PND.pdf

Guzmán, C. (2018). Avances y dificultades en la implementación del marco curricular común. Telebachillerato comunitario, educación Media superior a distancia y Telebachillerato estatal. México: INEE. 
Instituto Nacional de Estadística y Geografía INEGI. (2020). Información por Entidad.

Recuperado de http://cuentame.inegi.org.mx/monografias/informacion/pue/default.aspx?tema=me\&e $=21$

INEE. (2010). La educación media superior en México. Informe 20102011. México: INEE.

INEE. (2019). La educación obligatoria en México. Recuperado de https://www.inee.edu.mx/medios/informe2019/stage_01/ tem_05.html

Latapí, P. (2009). El derecho a la educación, su alcance, exigibilidad y relevancia en la política. RMIE [online], 14(40), 255-287.

Martínez Rizo, F. (2001). Las políticas educativas mexicanas antes y después del 2001. Revista Iberoamericana de educación, (27), 35-56.

Municipio de Tehuitzingo. (2021). Educación Media Superior en Tehuitzingo - Puebla. Recuperado de https://escuelasmex.com/ educacion-media-superior/municipio/tehuitzingo

Murphy, M. (2014). Self-determination as a collective capability: The case of indigenous peoples. Journal on human development and capability, 320-334. DOI: http://www.tandfonline.com/ doi/pdf/10.1080/19452829.2013.878320.

RIEMS. (2008). Reforma Integral para la Educación Media Superior. Recuperado de http://www..edu.mx/portal/docentes/ descargas/reforma_integral_educacion_media_superior_ riems.pdf

Sandoval, R. (2017). Territorialidad y contextos socioculturales para los procesos educativos de los jóvenes y adultos. The Biologist, 15(1), 186. DOI: https://doi.org/10.24039/rtb2017151154

SEP. (2012). Principales cifras 2012-2013. México: SEP. Recuperado de https://www.planeacion.sep.gob.mx/Doc/estadistica_e_indicadores/principales_cifras/principales_cifras_2012_2013. pdf

SEP. (2018). Adopción del modelo educativo para la educación obligatoria en el telebachillerato comunitario. Documento base 2018. México: SEP. Recuperado de https://www.dgb.sep.gob. $\mathrm{mx} /$ servicios-educativos/telebachillerato/normatividad/ Documento-base-2018.pdf 
SEP. (2019). Principales cifras del sistema educativo nacional 20182109. Recuperado de https://www.planeacion.sep.gob.mx/ Doc/estadistica_e_indicadores/principales_cifras/principales_cifras_2018_2019_bolsillo.pdf

SEP. (2019). Principales cifras del sistema educativo nacional 20192120. Recuperado de https://www.planeacion.sep.gob.mx/ Doc/estadistica_e_indicadores/principales_cifras/principales_cifras_2018_2019_bolsillo.pdf

Soberanes, J. (2015). Las reformas constitucionales. México: Universidad Nacional Autónoma de México.

Solís, P. (2018). La transición de la educación secundaria a la educación media superior en México: el difícil camino a la cobertura universal. Perfiles educativos, 40(159).

Subsecretaria de Educación Media Superior. (2008). Educación Media Superior. Recuperado de http://educacionmediasuperior. sep.gob.mx/

Subsecretaria de Educación Media Superior. (2008). Sistema Nacional de Bachillerato. Recuperado de http://www.sems.gob.mx/ es/sems/sistema_nacional_bachillerato

Tapia, L. A. y Valenti, G. (2016). Desigualdad educativa y desigualdad social en México. Perfiles educativos, 38(151).

Villa Leve, L. (2014). La educación media superior en México. Jóvenes y desigualdad de oportunidades. Revista Innovación Educativa México, 14(64).

Weiss, E. (2012). La educación media superior en México ante el reto de su universalización. Archivos de Ciencias de la Educación, 6(6), 4a época. Recuperado de http://www.archivosdeciencias.fahce.unlp.edu.ar/ 